Original Research Paper

\title{
Can Grammar Define Similarity of Human Natural Languages?
}

\author{
${ }^{1}$ Vladimir Nikolaevich Polyakov, ${ }^{2}$ Ivan Sergeevich Anisimov and ${ }^{3}$ Elena Andreevna Makarova

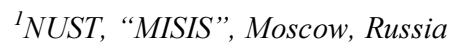 \\ 2 "Yandex", LLC, Moscow, Russia \\ ${ }^{3}$ Institute of Linguistics of RAS, Moscow, Russia
}

Article history

Received: 11-06-2016

Revised: 03-10-2016

Accepted: 05-09-2016

Corresponding Author:

Vladimir Nikolaevich Polyakov

NUST "MISIS", Moscow,

Russia

Email: pvn-65@mail.ru

\begin{abstract}
The aim of the present study is to show that similarity of human natural languages can be conveyed not only by phonetic data, but also by grammar. The paper regards the largest typological database WALS and its possibilities in the sphere of genealogic relationship of languages. Using the method of two-objective optimization and data mining, which is new for linguistic studies, we show that grammatical (structural) data, as well as phonetic data, can deliver information on the similarity of languages. Language isolates and micro-families do not have genealogic relatives based on phonetic information, but they do have genealogic relatives based on grammar information.
\end{abstract}

Keywords: WALS, Two-Objective Optimization, Data Mining, Language Isolates, Micro-Families, Similarity, Grammar

\section{Introduction}

The main source of information for the present study is World Atlas of Language Structures-WALS (Haspelmath et al., 2005). It is the world's biggest database describing structural properties of languages. The first version of the database appeared as a book and a CD with a stand-alone application "Interactive Reference Tool (WALS Program)". WALS Program contains description for 2,560 languages according to 142 features. The online version of the database (Dryer and Haspelmath, 2013) was published in 2011. Now, it describes 2,679 languages according to 144 features (Comrie et al., 2013) and some of the features are divided into several parts each including a separate map and set of languages.

The core of WALS is made by chapters dedicated to features that are divided into eight main areas encompassing the major structural domains of grammar: Phonology, morphology, nominal categories, nominal syntax, verbal categories, word order, simple clauses and complex sentences. There are also smaller areas describing lexicon, sign languages, writing systems and paralinguistic usage of clicks.

WALS contains 160 maps showing the geographical distribution of the features. Besides, the program allows combining up to four different features on a single map, which can be useful for studying correlation of features.

Full grammar books, dictionaries, dissertations, articles, field notes, questionnaires and authors' own knowledge became the source of information. WALS, being the result of work of 55 specialists, was the first feature atlas on a world-wide scale, which made it possible to compare unrelated languages all over the world.

The present study uses the data WALS in an attempt to solve the problem that has been pending for many decades, if not a hundred years, -classification of language isolates. A language isolate does not have any demonstrable genealogic relationship with any existing language, i.e., the traditional methods of comparative linguistics based on the comparison of basic concepts (100-word lists) (Swadesh, 1952) were unable to define genealogic relations between a language isolate and any other languages.

Many linguists believe that all languages on our planet had originated from a single mother tongue, the reason why the problem of classifying language isolates has become the object of numerous studies (Tallerman and Gibson, 2012).

In contrast to unclassified languages, which are likely to demonstrate a genealogic relationship with some family once they are studied further, language isolates have already been thoroughly described, but they still do not classify with any known language family.

In 1939 Prince Trubetzkoy defined six grammar features characteristic of all Indo-European languages (Trubetzkoy, 1939). Over a decade later, Emile Benveniste started an indirect dispute with him in (Benveniste, 1954). He claimed that one of the languages 
of North-American Indians-Takelma-possessed all six grammar features indicated by Trubetzkoy. But Takelma was a language isolate (i.e., it did not have any genealogic relatives), moreover, it was located on a different continent from speakers of Indo-European languages. Thus, there was no way Takelma could be a representative of Indo-European languages. We shall return to this question in the Discussion section.

In the present paper, we suggest using the methods of two-objective optimization (Ehrgott, 2000; Izraylevich and Tsudikman, 2012) for pair-wise comparison of typological language profiles. This might be able to cast light on the genealogic relationship of some language isolates, a question that classic methods of comparative linguistics failed to answer.

In addition, WALS has undergone data cleaning in style of data mining so that only structural features are compared. It allows finding a distinct border between well described and poorly described pairs of languages. In the future, if more information is added to WALS, the problem of classifying language isolates and microfamilies can be successfully solved.

The present research is compared to a seemingly similar study, conducted by Wichmann and Holman (2010), but, as shown below, the method and the results presented in this study, are quite different (cf. Section 4).

Approaches concerning the scenarios of evolution basing on WALS features were used in works of Gray et al. (2011).

\section{Materials and Methods}

\section{Two-Objective Optimization}

The present study uses the method of two-objective optimization, first described in (Edgeworth, 1881). This method approved itself in computer science (Ehrgott, 2000) and is widely used for solving economic and technical problems (Izraylevich and Tsudikman, 2012).

The following two criteria are considered: The percentage of matching features values from WALS Program and the number of coinciding features. The point is that if only the percentage of matching values of features is considered, there is likely to be a language with the number of described features equaling, for example, 2 and their values will both match the values of the language under study. In order to exclude such unreliable data, the second parameter-the number of coinciding features is introduced. It was experimentally established to be 30 , while the percentage of matching values must be at least $65 \%$.

In two-objective optimization, one criterion is chosen as the main one and the other becomes subsidiary. In this study, the main criterion is the percentage of matching values of features and the restricting criterion is the number of coinciding features. The latter is restricted from below (language under study and structurally maximally close languages must have at least 30 coinciding features); and the former tends to reach $100 \%$, but must be at least $65 \%$.

\section{Database "Isolates"}

The information for the creation of the database "Isolates" (which is used for search of Structurally Maximally Close languages (SMC-languages) was taken from WALS Program, as it is available for download and presented in form of an electronic database, which simplifies computer analysis of the information. The data necessary for the present study include list of languages, list of features and the descriptions of the languages according to the features.

\section{Description of the Method}

The aim of the method is to find a language that has the highest percentage of matching values of features for any language under study and, at the same time, meets the requirements, i.e., the number of coinciding features is at least 30 and the percentage of matching values is at least $65 \%$. The given choice of parameters will be discussed further.

MS Access, being the easiest and the most accessible tool, is chosen to be used in this study. Four queries will be needed: QUERY-1, QUERY-2, QUERY-3 and QUERY-4.

QUERY-1 (Fig. 1) allows getting a complete list of languages, features and values of grammar features. A total of 58,019 entries (according to WALS Program) were received.

QUERY-2 (Fig. 2) allows getting from the database a list of languages and an integrated number of their features whose values match the values of the language under study. The list is sorted by the decrease in the number of matches. QUERY-2 is built by uniting two queries QUERY-1.

QUERY-3 (Fig. 3) allows getting a list of languages and an integrated number of their features from the database whose values do not match the values of the language isolate. The list is sorted by the decrease in the number of mismatches.

QUERY-3 is built by uniting two queries QUERY-2.

QUERY-4 allows getting from the DB "Isolates" a list of languages and an integrated number of their features whose values match the values of the language isolate. The list is sorted by the decrease in the number of matches. The query cuts off the results with the total number of coinciding features below 30 and sorts the search results from the highest percentage of matching values (Fig. 4). QUERY-4 is built by uniting two queries: QUERY-2 and QUERY-3. 


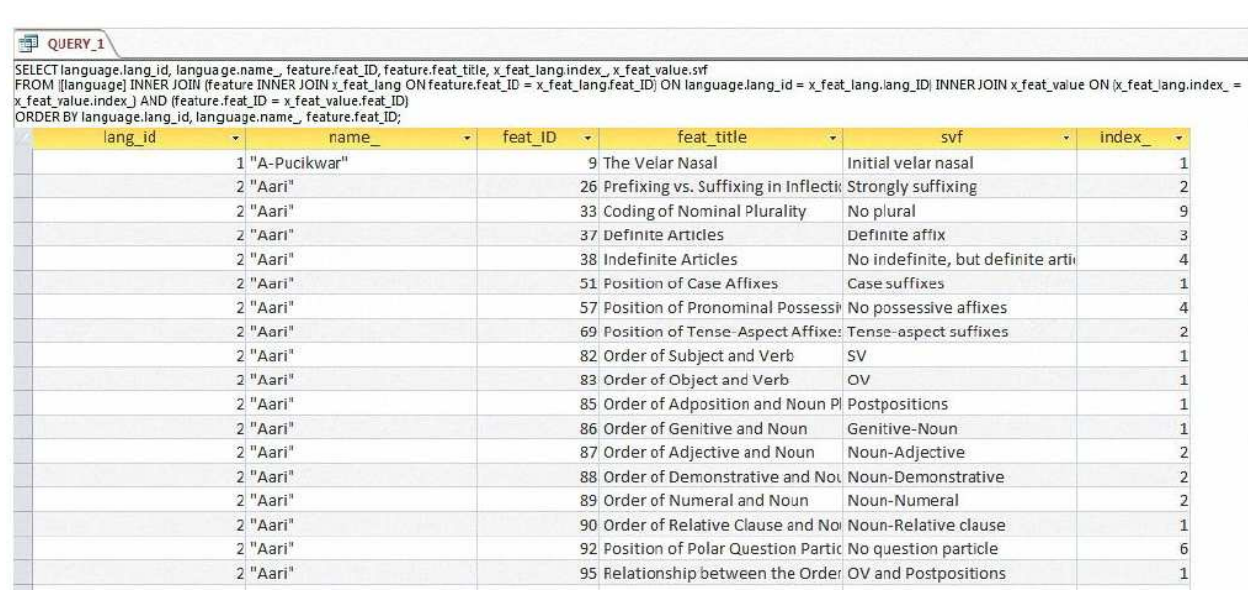

Fig. 1. QUERY-1

\begin{tabular}{|c|c|c|c|c|c|c|}
\hline 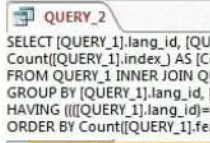 & 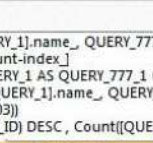 & 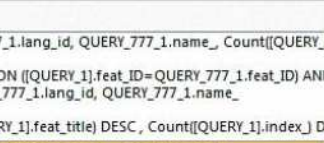 & 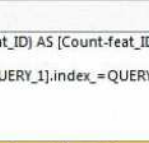 & & $\begin{array}{l}\text { unt[[QUERY_1].feat_title } \\
\text { 1.index_J }\end{array}$ & AS [Count-feat_titite], \\
\hline QUERY_1.lang_id & - QUERY_1.na. & QUERY_TI. QUERY_TII_1.name_. & Count-feat_ID & - & Count-feat_title - & Count-inde: : \\
\hline & 703 "German" & 703 "German" & & 129 & 129 & 129 \\
\hline & 703 "German" & 655 "French" & & 91 & 91 & 91 \\
\hline & 703 "German" & 626 "English" & & 90 & 9 & 90 \\
\hline & 703 "German" & 1952 "Russian" & & 87 & 87 & 87 \\
\hline & 703 "German" & 742 "Greek (Modern)" & & 77 & 7 & 77 \\
\hline & 703 "German" & 2089 "Spanish" & & 75 & 75 & 75 \\
\hline & 703 "German" & 647 "Finnish" & & 72 & 72 & 72 \\
\hline & 703 "German" & 849 "Hungarian" & & 69 & 69 & 69 \\
\hline & 703 "German" & 1251 "Latvian" & & 64 & 64 & 64 \\
\hline & 703 "German" & 1008 "Kannada" & & 61 & 61 & 61 \\
\hline & 703 "German" & 825 "Hindi" & & 58 & 58 & 58 \\
\hline & 703 "German" & 2311 "Turkish" & & 55 & 55 & 55 \\
\hline & 703 "German" & 816 "Hebrew (Modern)" & & 55 & 55 & 55 \\
\hline & 703 "German" & 1155 "Korean" & & 53 & 53 & 53 \\
\hline & 703 "German" & 1836 "Persian" & & 53 & 53 & 53 \\
\hline & 703 "German" & 602 "Dutch" & & 52 & 52 & 52 \\
\hline & 703 "German" & 1270 "Lezgian" & & 50 & 50 & 50 \\
\hline & 703 "German" & 109 "Apurinã" & & 50 & $5 c$ & 50 \\
\hline
\end{tabular}

Fig. 2. QUERY-2

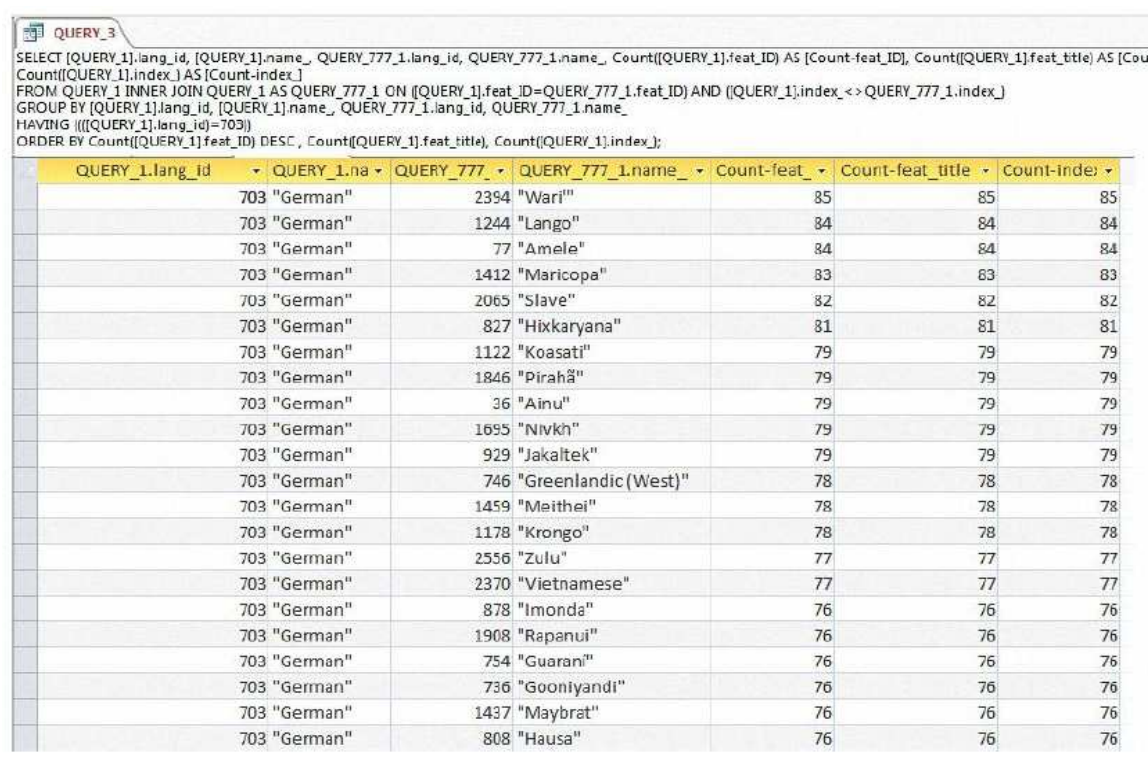

Fig. 3. QUERY-3 


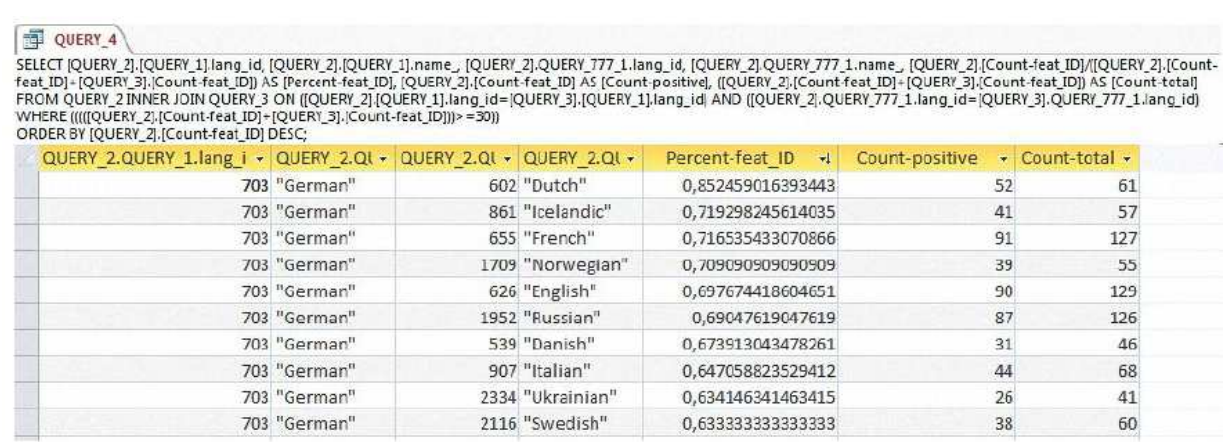

Fig. 4. QUERY-4

Table 1. Choice of parameters for the formal method of search for SMC-languages

\begin{tabular}{lllll}
\hline Family & Language & $\begin{array}{l}\text { Range of percentage match } \\
\text { of values in features }\end{array}$ & $\begin{array}{l}\text { Range of the number of } \\
\text { common features }{ }^{1}\end{array}$ & $\begin{array}{l}\text { Number of similar } \\
\text { languages }^{2}\end{array}$ \\
\hline Altaic & Turkish & $86.8-87.8 \%$ & $38-41$ & 2 \\
& Turkmen3 & - & - & - \\
\multirow{3}{*}{ Uralic } & Tuvan & $81.8-90.6 \%$ & $32-51$ & 7 \\
& Estonian & $76.7-79.2 \%$ & $30-48$ & 2 \\
\multirow{5}{*}{ Indo-European } & $79.2-80.5 \%$ & $41-48$ & 2 \\
& Finnish & $76.7-80.5 \%$ & $30-41$ & 2 \\
& Saami (Northern) & $71.1-96.7 \%$ & $30-49$ & 7 \\
& Serbian-Croatian & $71.1-83.1 \%$ & $32-128$ & 6 \\
& Spanish & $64.6-95.3 \%$ & $31-65$ & 10 \\
\hline
\end{tabular}

Notes: 1) The admission was at the level of 30. When the value was lower, the result of the search was inconclusive.

2) Number of similar languages until the first non-relative language appeared.

3) For Turkmen WALS program does not contain enough grammar descriptions that satisfy the requirements of our method.

\section{Choice of Parameters}

The parameters of two-objective optimization (at least 30 features and $65 \%$ of matching values) have been defined above, but the method has not been specified. The first approach was empirical-a result of numerous experiments. Later, a formal method of defining the boundaries (30 features and $65 \%$ ) will be suggested (cf. below).

The table contains data on 9 languages from 3 families of languages (Altaic, Uralic, Indo-European). The similarity of these languages is confirmed by phylogenetic tree on the base of lexical and phonetic data (Wichmann et al., 2013). Table 1 shows the choice of parameters ( $\%$ of matching values and number of common features) for the formal method of search for SMC-languages.

Formal Description of the Two-Objective Optimization Method Used for Problem Solution

Problem of search for the Structurally Maximally Close Language (SMCL) $j$ for any Language under Study (LUS) $i$

Let:

- $L=\left\{l_{1}, l_{2} \ldots, l_{i}, \ldots l_{n}\right\}$ be a set of languages, be the percentage of matching feature values for languages $i$ and $j$
- $\quad P_{i j}^{\text {match }}$ be the number of coinciding features for languages $i$ and $j$

We have a language $i$. The problem is to find its maximally close language $j$ that belongs to set $L\left(l_{j} \in L\right)$. We will solve the problem of two-objective optimization (Ehrgott, 2000:17) by choosing one criteria as the main one similar to (Izraylevich and Tsudikman, 2012:79):

- We choose $P_{i j}^{\text {match }}$ as the main criterion

- $\quad N_{i j}^{\text {coinc }}$ Then becomes the restricting criterion

Thus, the solution of the problem comes down to search for language $j^{\text {opt }}$ that has the maximal percentage of matches $P_{i j}^{\text {match }}$ with the given restriction $N^{\text {coinc }-l i m}$ for the number of coinciding features $N_{i j}^{c o i n c}$ for the given language $i$ and languages $j(j=1 \ldots n)$ :

$$
j^{\text {opt }}=\operatorname{argmax}\left(P_{i j}^{\text {match }}\right), N_{i j}^{\text {match }} \geq N^{\text {coinc_lim }}, j=1 \ldots n
$$

Where:

$$
P_{i j}^{\text {match }}=\frac{N_{i j}^{\text {match }} * 100}{N_{i j}^{\text {coinc }}}
$$


$N_{i j}^{\text {match }}$ is the number of matching feature values of the languages.

\section{Criticism of WALS Descriptions and Data Mining}

The results received by the method described above seemed to be very encouraging, but still needed additional verification. Moreover, they were criticized by typologists for the fact that using the whole set of features from WALS in the two-objective optimization, we considered some non-structural features. (The information was anonymous, received as critical notes from one of the linguistic journals).

Thus, the verity of the data that can be received using the formal method of search for SMC-languages was checked on a so-called "Test A". Test A included all languages from WALS Programs that begin with the letter "A". Unfortunately, the results were unsatisfactory: SMC-languages belonged to the same family as the language under study only in 15 out of 35 cases, i.e., $42.9 \%$. The data on the classification of a language with a certain language family was taken from WALS Online. The results of Test A are presented in Table 2.

Such results are believed to be accounted for by the fact that WALS does not have enough description for all languages. Besides, it includes such nongrammatical features as "lexicon", for instance, which can easily be borrowed by languages due to areal contacts, besides the features describing the vocabulary of the languages, there is an area "sign language" and values "other" and "not reported", which do not necessarily mean that these values actually match.

Table 2. Results of Search for SMC-languages for test "A"

\begin{tabular}{|c|c|c|c|c|c|c|}
\hline $\begin{array}{l}\text { Language } \\
\text { under study }\end{array}$ & $\begin{array}{l}\text { Family of language } \\
\text { under study }\end{array}$ & SMC-language & $\begin{array}{l}\text { Family of } \\
\text { SMC-language }\end{array}$ & $\begin{array}{l}\text { Number of } \\
\text { coinciding } \\
\text { features }\end{array}$ & $\begin{array}{l}\% \text { of } \\
\text { matching } \\
\text { values }\end{array}$ & $\begin{array}{l}\text { Same } \\
\text { family }\end{array}$ \\
\hline Abipón & Guaicuruan & Achumawi & Hokan & 32 & $71.90 \%$ & - \\
\hline Abkhaz & Northwest Caucasian & Kolami & Dravidian & 35 & $71.40 \%$ & - \\
\hline Acehnese & Austronesian & Chrau & Austro-Asiatic & 39 & $64.10 \%$ & - \\
\hline Acehnese & Austronesian & Chrau & Austro-Asiatic & 39 & $64.10 \%$ & - \\
\hline Acoma & Keresan & Luiseño & Uto-Aztecan & 34 & $64.70 \%$ & - \\
\hline Akan & Niger-Congo & Irarutu & Austronesian & 32 & $68.80 \%$ & - \\
\hline Alamblak & Sepik & Kiwai & Kiwaian & 38 & $76.30 \%$ & - \\
\hline Alawa & Mangarrayi-Maran & Nunggubuyu & Gunwinyguan & 38 & $84.20 \%$ & - \\
\hline Albanian & Indo-European & Romanian & Indo-European & 56 & $76.70 \%$ & + \\
\hline Alyawarra & Pama-Nyungan & Kanuri & Saharan & 32 & $78.10 \%$ & - \\
\hline Amahuaca & Panoan & Shipibo-Konibo & Panoan & 34 & $73.50 \%$ & + \\
\hline Amele & Trans-New Guinea & Barai & Trans-New Guinea & 33 & $90.90 \%$ & + \\
\hline Amharic & Afro-Asiatic & Marathi & Indo-European & 40 & $75.00 \%$ & - \\
\hline Anejom & Austronesian & Tigak & Austronesian & 34 & $70.60 \%$ & + \\
\hline Angas & Afro-Asiatic & Yulu & Central Sudanic & 33 & $81.80 \%$ & - \\
\hline Ao & Sino-Tibetan & Usan & Trans-New Guinea & 31 & $77.40 \%$ & - \\
\hline Apalaí & Cariban & Hixkaryana & Cariban & 43 & $76.70 \%$ & + \\
\hline Apinayé & Macro-Ge & Canela-Krahô & Macro-Ge & 30 & $90.00 \%$ & + \\
\hline Apurinã & Arawakan & Seneca & Iroquoian & 39 & $74.30 \%$ & - \\
\hline Arabic (Egyptian) & Afro-Asiatic & Arabic (Moroccan) & Afro-Asiatic & 39 & $84.60 \%$ & + \\
\hline Araona & Tacanan & Tacana & Tacanan & 38 & $71.10 \%$ & + \\
\hline Arapesh & Torricelli & Tiwi & Tiwian & 93 & $68.80 \%$ & - \\
\hline Arawak & Arawakan & Baré & Arawakan & 30 & $66.70 \%$ & + \\
\hline Archi & Nakh-Daghestanian & Avar & Nakh-Daghestanian & 42 & $85.70 \%$ & + \\
\hline Armenian (Eastern) & Indo-European & Brahui & Dravidian & 67 & $68.70 \%$ & - \\
\hline Armenian (Western) & Indo-European & Lezgian & Nakh-Daghestanian & 31 & $67.70 \%$ & - \\
\hline Arosi & Austronesian & Indonesian & Austronesian & 30 & $66.70 \%$ & + \\
\hline Arrernte & Pama-Nyungan & Wahgi & Trans-New Guinea & 32 & $78.10 \%$ & - \\
\hline Asmat & Trans-New Guinea & Shiriana & Yanomam & 35 & $74.30 \%$ & - \\
\hline Atayal & Austronesian & Hawaiian & Austronesian & 31 & $64.50 \%$ & + \\
\hline $\mathrm{Au}$ & Torricelli & Bagirmi & Central Sudanic & 30 & $70.00 \%$ & - \\
\hline Avar & Nakh-Daghestanian & Tsova-Tush & Nakh-Daghestanian & 33 & $93.90 \%$ & + \\
\hline Awa Pit & Barbacoan & Mari (Meadow) & Uralic & 35 & $71.40 \%$ & - \\
\hline Awtuw & Sepik & Uradhi & Pama-Nyungan & 35 & $80.00 \%$ & - \\
\hline Aymara (Central) & Aymaran & Jaqaru & Aymaran & 45 & $80.00 \%$ & + \\
\hline Azerbaijani & Altaic & Turkish & Altaic & 41 & $87.80 \%$ & + \\
\hline
\end{tabular}


Thus, it was decided to mark the following features and values as "insignificant" and exclude them from the queries:

- All features from the area "lexicon" (features 129138)

- All features from the area "sign languages" (features 139-140)

- Values "other" and "not reported" from features 5 ("Voicing and Gaps in Plosive Systems"), 24 ("Locus of Marking in Possessive Noun Phrases"), 74 ("Situational Possibility"), 75 ("Epistemic Possibility"), 95 ("Relationship between the Order of Object and Verb and the Order of Adposition and Noun Phrase"), 96 ("Relationship between the Order of Object and Verb and the Order of Relative Clause and Noun"), 97 ("Relationship between the Order of Object and Verb and the Order of Adjective and Noun"), 142 ("ParaLinguistic Usages of Clicks")

Query-1 was changed so that only values that are not marked as "insignificant" are taken into consideration. Due to the smaller total number of values, it was decided to lower the minimal number of coinciding features to 26 .

The total number of languages, for which an SMC language was found, equals 523. As for the other languages from WALS Program, they are either poorly described or the SMC-language had the percentage of matching features below the limit.

This procedure reminds the stage of data cleaning within the framework of the data mining theory, but, at the same time, it has the specificity of linguistic typology. One of the earliest works in the sphere of data mining is presented in (Fayyad et al., 1996). Thus, after the cleaning, it is believed that the structural features from WALS were kept for further research.

\section{P-Pairs and G-Pairs of Languages}

Comparative linguistics aimed at establishing the historical relatedness of languages compares their phonological and morphological systems, lexicon and syntax (Bynon, 1977), but sometimes it can only consider the lists of basic concepts-Swadesh lists (Swadesh, 1952).

A striking example of such method is Automated Similarity Judgment Program (ASJP) (Wichmann et al., 2013)-a project whose major goal is to classify all languages of the world applying computational approaches to comparative linguistics. Other purposes of the program include: Determining the homeland of a protolanguage, evaluating phylogenetic methods, investigating sound symbolism and a few more. Besides the universally recognized language families, the database includes language isolates, creoles, pidgins, mixed languages and constructed languages.

The similarity of languages is calculated automatically by using edit distance (Levenshtein, 1966). Originally, ASJP used 100-word lists, but later the authors came to a conclusion that shorter lists, containing only 40 words, gave the same accurate results. The work on the project is still going on and the number of languages (i.e., lists of words) is constantly being expanded.

The pairs of languages, whose genealogical similarity has been proven by comparative linguistics and ASJP and recognized by the linguistic society, shall be called P-pairs (Phonetic pairs). In order to avoid discrepancy due to different transcription systems and in order to embrace languages that do not have a writing system, Swadesh method, comparative linguistics and ASJP compare phonetic images of the words denoting the basic concepts (40 or 100 word lists); the reason why Ppairs of languages are referred to those pairs of genealogically related languages, whose similarity is stabled by ASJP method.

On the other hand, pairs of languages found by the method of two-objective optimization and data-mining described in this study present grammatically closest languages without considering lexicon at all, thus the term G-pairs (Grammatical pairs) of languages will be applied for them.

Pairs of languages that are recognized to be members of the same family and that present structurally closest languages will be called PG-languages.

Figure 5 shows a diagram for all 523 languages from WALS Program, for which a SMC-language was found. G-pairs, where the language under study and its SMC language do not belong to the same family, are marked with white circles. Black squares mark PG-languages, i.e., G-pairs where languages belong to the same family. The diagram does not contain language isolates.

It can be seen that the more features of two languages coincide and the more values match, the higher the possibility that a G-pair is also a P-pair.

A line can be drawn through the four white circle. These are the four G-pairs of languages with the highest percentage of matching values. All languages above the line are PG-languages. As for the languages below the line, they can be either G-languages or PG-languages. In further studies, the view of this line can be specified due to the expansion of language descriptions.

We believe that the genealogic relatedness of languages proven by lexical data must also be reflected in their grammatical structure. Judging from the diagram, it stands true for the languages above the line.

As WALS is replenished with more information, this conclusion will become more evident. 


\section{Distribution of pairs "Language isolate and its SMC-language" (based on WALS Program)}

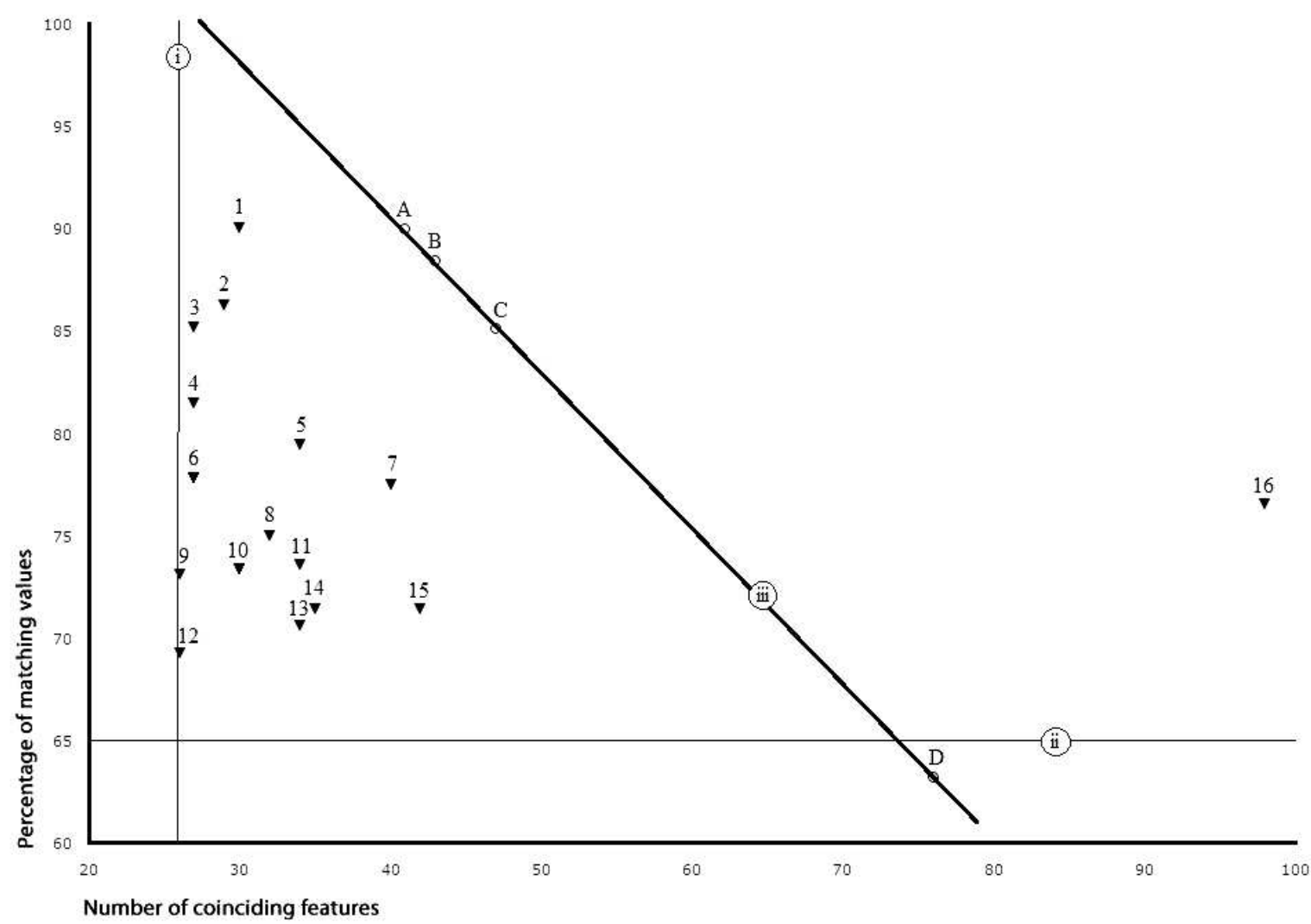

Lines:

$\mathrm{i}$ - line designating the minimal number of coinciding features equaling 26

ii - line designating the minimal percentage of matching values equaling $65 \%$

iii - line separating the area of PG-pairs of languages. Drawn through 4 points (A, B, C, D) marking G-pairs of languages

Languages:

1 - Korean - Kalmyk (Altaic)

2 - Burushaski - Karachay-Balkar (Altaic)

3 - Sulka - Luvale (Niger-Congo)

4 - Warao - Fasu (Trans-New Guinea)

5 - Ainu - Kiwai (Kiwaian)

6 - Basque - Digaro (Sino-Tibetan)

Tonkawa - Fulniô (Yatê)

Tunica - Kunimaipa (Trans-New Guinea)

7 - Waorani - Suena (Trans-New Guinea)

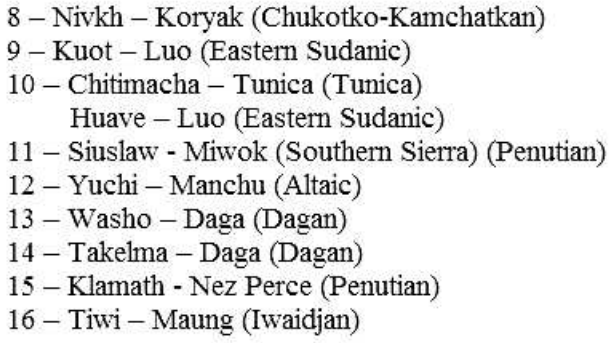

Fig. 6. Diagram of distribution of pairs "Language isolate \& its SMC-language" relatively to the area of PG-languages

Nevertheless, one pair of languages lies above the line-Tiwi and Maung. Thus, it can be claimed that these two languages are relative with Tiwi belonging to Iwaidjan family, where Maung is classified with.

Additionally, the pair of languages in question also meets the requirements suggested by Wichmann and Holman (2010). The authors compared profiles of languages presented in WALS pairwise and set the boundary of 45 coinciding features and $81.5 \%$ of matching values.
Despite the seeming similarity of the approaches, it should be stressed that in the present study, a descending line as a boundary between the area that includes only PG-languages and the area of both Ppairs and G-pairs of languages is suggested. It means that the more coinciding features a pair of languages has, the lower can the percentage of their matching values for the languages to appear in the area of PGlanguages be. 
Table 3. Query results for 19 language isolates

\begin{tabular}{llll}
\hline $\begin{array}{l}\text { Language isolate } \\
\text { (Language under study) }\end{array}$ & SMC-language & $\begin{array}{l}\text { Number of matching } \\
\text { features }\end{array}$ & $\begin{array}{l}\text { Percentage of matching } \\
\text { values in WALS program }\end{array}$ \\
\hline Ainu & Kiwai & 34 & $79.41 \%$ \\
Basque & Digaro & 27 & $77.78 \%$ \\
Burushaski & Karachay-Balkar & 29 & $86.21 \%$ \\
Chitimacha & Tunica & 30 & $73.33 \%$ \\
Huave & Luo & 30 & $73.33 \%$ \\
Klamath & Nez Perce & 42 & $71.43 \%$ \\
Korean & Kalmyk & 30 & $90 \%$ \\
Kuot & Luo & 26 & $73.08 \%$ \\
Nivkh & Koryak & 32 & $75 \%$ \\
Siuslaw & Miwok (Southern Sierra) & 34 & $73.53 \%$ \\
Sulka & Luvale & 27 & $85.19 \%$ \\
Takelma & Daga & 35 & $71.43 \%$ \\
Tiwi & Maung & 98 & $76.53 \%$ \\
Tonkawa & Fulniô & 27 & $77.78 \%$ \\
Tunica & Kunimaipa & 27 & $77.78 \%$ \\
Waorani & Suena & 40 & $77.5 \%$ \\
Warao & Fasu & 27 & $81.48 \%$ \\
Washo & Daga & 34 & $70.59 \%$ \\
Yuchi & Manchu & 26 & $69.23 \%$ \\
\hline
\end{tabular}

On the other hand, the whole set of phonetic information (40-item word lists of ASJP, inflexional affixes and endings, prepositions, etc.) does not present any prove of the similarity of these two languages. The question is: How Tiwi and Maung managed to preserve similarity in grammar, but not in phonetics. The possibility of their resemblance due to borrowings is discharged, as they are structurally maximally close languages.

\section{Micro-Families}

Micro-families is a term introduced by the authors of the present article. Micro-families are separate families of languages including up to 10 languages. Microfamilies are claimed to be similar to language isolates. The difference is that due to a number of evolutionary and historical reasons, the linguistic diversity of microfamilies is broader than that of language isolates. Another factor is their wider area of distribution and presence of dialects, which indicate more favorable historical conditions. Consequently, there is a reason for searching a relative family for micro-families like for language isolates.

In this study, 35 languages from 25 micro-families (the number of micro-families that have at least one language with enough features described) were studied. As a rule, there were 1-2 such languages in each family. These languages were taken as languages under study and a language from a different family and with the highest percentage of matching values was chosen as their SMC-language. The results are shown in Fig. 7.

Unfortunately, the grammar information in WALS is insufficient for a reliable conclusion on the genealogical relationship of micro-families (all pairs of languages on the diagram are below the line iii).

\section{Results}

The method of SMC-language gave hope for establishing the connection between a language isolate and its relative. This idea suggests the following hypotheses.

First, grammatical, or to be more precise, structural data, as well as phonetic data, can deliver information on the genealogic similarity of languages.

Second, language isolates and micro-families do not have genealogic relatives based on phonetic information (P-pairs), but they do have genealogic relatives based on grammar information (G-pairs).

Third, the results for the language isolate Tiwi and its SMC-language Maung from Iwaidjan micro-family allowed us suggesting a hypothesis that these two languages are genealogically related.

\section{Discussion}

It can be concluded that grammar information is an important source of data about the relationship of languages, as phonology, morphology and lexicon.

WALS is the world's biggest typological database, whose first version-WALS Program-was used as the basis for the present study. We used all the information presented in WALS, which is at the present time the most complete collection of typological data on the languages. Realizing that these data are still not enough for reliable theories, we suggest hypotheses hoping that further replenishment of WALS will prove them. Now WALS contains the description of 2,560 languages. But only 523 of these languages (about 20.4\%) have structure description sufficient for communication of information about genealogic relationship. 


\section{Distribution of pairs "A representative of a micro-family and its SMC-language"}

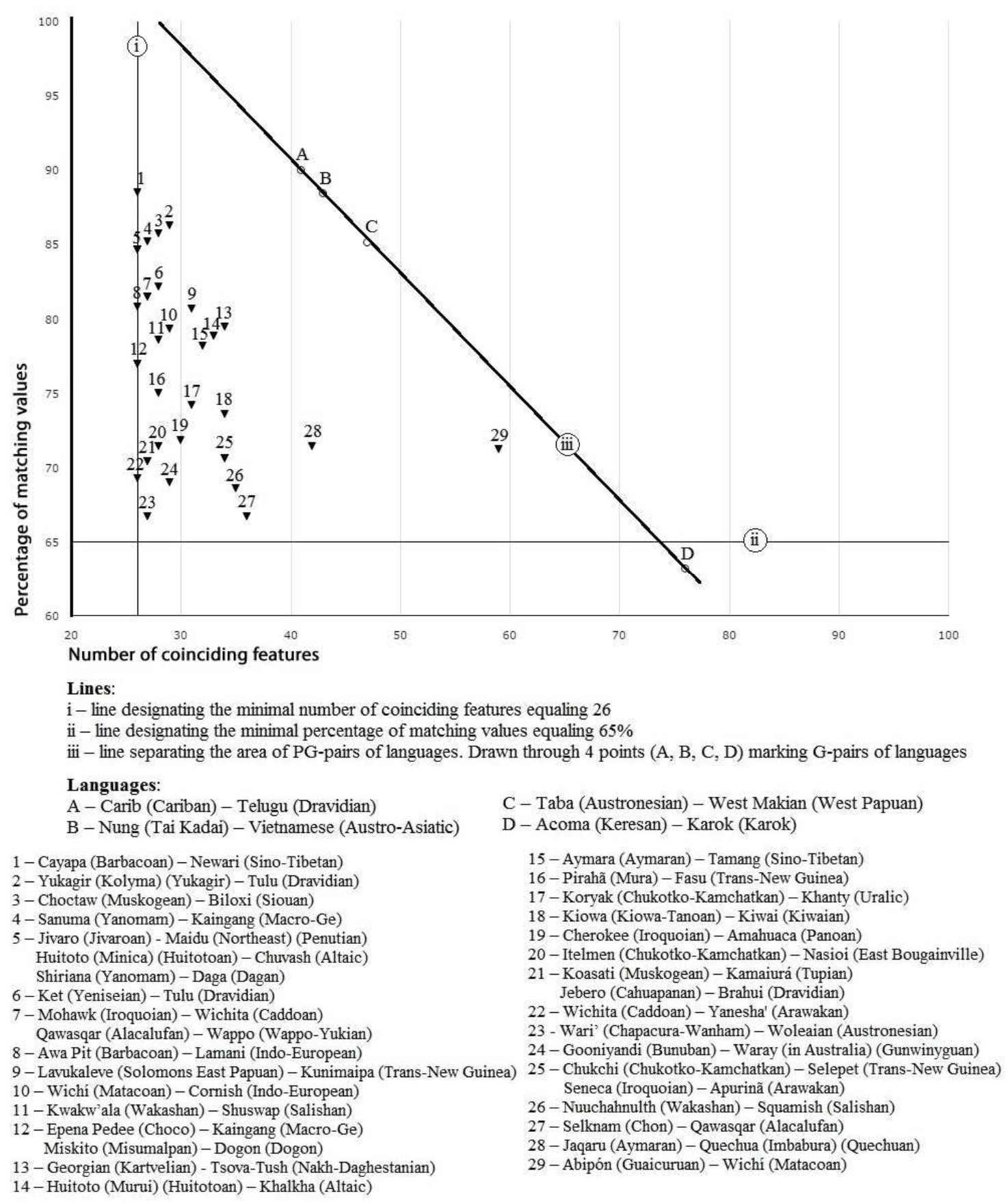

Fig. 7. Distribution of pairs "A representative of a micro-family \& its SMC-language" relatively to the area of PG-languages, Note: If one number designates over one pair, it means that the number of coinciding features and the percentage of matching values were the same for these pairs of languages.

Therefore, it is necessary to replenish WALS with more information about languages, will which make the database even more important and useful tool for further studies.

Optimization methods are widely used in all spheres; they are diverse and acknowledged to be part of computer science (Ehrgott, 2000). In linguistic typology the method of optimization was used for the first time. We decided on the variant of two-objective optimization as it best suits for the nature of the data. As for statistics, we believe that the method of optimization seems more 
precise, though we would like to state that we are not criticizing mathematics statistics in any way.

We used a SQL-like languages (language of MS Access), as SQL is de facto the standard in the sphere of informational databases (Codd, 1970).

The article contains all queries for the database "Isolates" in order to make them easier to reproduce in case the queries (Fig. 1-4), the method of two objective optimization and the graphs (Fig. 5-7) need additional verification, as well as the conclusions. Moreover, the queries can be reproduced using any relational DBMS. The database "Isolates" is a slightly changed fragment of WALS Program. All data from WALS Program (Haspelmath et al., 2005) and WALS Online (Dryer and Haspelmath, 2013) are available in free access.

One of the goals of the present research was an attempt to classify language isolates using a new formal method, based on the structural information about languages. A similar attempt, though proposing a different aim, was made by Wichmann and Holman (2010) "Pairwise Comparison of Typological Profiles". The authors of the article established the boundaries separating the area of languages belonging to the same families from the area that does not guarantee a necessary genealogic relationship of languages to be at least 45 coinciding features and $81.5 \%$ of matching values.

In contrast to their research, we believe that these numbers can be lowered provided that the comparison of typological profiles excludes non-structural features, as well as values that do not necessarily mean a match, e.g., value "other". The minimal number of coinciding features can be 26 and the lowest percentage of matching values is $65 \%$. Moreover, the line indicating the border between the pairs of languages that belong to the same family and the area that includes both G-pairs and Ppairs of languages should be descending. It is conditioned by the fact that the increase of the number of coinciding features decreases the probability of accidental match of a set of values.

The positive result of search of SMC-languages for language isolates included only one pair of languagesTiwi (a language isolate) and Maung (an Iwaidjan language). The other 18 pairs of languages fell below the boundary of the area of PG-languages. This can be accounted for by the fact that only $20.4 \%$ of all languages from WALS Program contain enough information for this study. Thus, once WALS supplements the other language profiles with more data, the application of the formal method for language isolates, as well as micro-families, will give more remarkable results.

We believe that there will be no dramatic change in the percentage of matching values for language isolates and their SMC-languages if WALS is replenished with more descriptions, but the G-pairs of languages will move further to the right of the diagram falling into the area of guaranteed similarity.
For many decades, scientists have been trying to find genealogic relations for language isolates using the whole amount of phonetic information. Hereby, not only limited lists of basic vocabulary, but also the accompanying information (case system (prepositions, endings, postposition, word-forming and inflectional affixes, conjunctions, pronouns, toponyms, onomasticon, names of animals and plants, etc.) is meant. However, there were no results in this sphere.

We showed that both authors (Benveniste and Trubetzkoy), whom we highly respect, were wrong in their own way. Their misbelief can be explained by the poor elaboration of grammar theory in the 20th century. Trubetzkoy did not have enough data to make conclusions on the similarity of Indo-European languages. Even at the present time genealogic features of a family are unlikely to be reliably defined by the frequency method (Makarova and Polyakov, 2015; Danilova et al., 2016). It is only possible to define the closest grammatical relative for a language. The theory and practice of grammar statistics still has to find a convincing answer for this question.

Benveniste was wrong in denying the possibility of classifying languages according to the genealogical principle basing on grammar data. It becomes more obvious that such genealogical classification can exist providing that there is enough structural data on the languages.

Emile Benveniste was right to claim that Takelma does not belong to the Indo-European languages. In the present research (Table 3) we show that the most probable genealogical relative (SMCL) for Takelma (LUS) is Daga from the Dagan family of languages (spoken in Papua New Guinea).

Although the formal method of search of SMC languages can help find genealogic relations between a pair of languages, it does not answer the question on the type of their connection-whether a SMC-language is parent or daughter of the language under study, or they both descended from a common ancestor (not described in WALS or lost historically).

In order to answer the question above we should study the historical context, analyze the possible direction of settlement on continents and archipelagos, compare the areas of language under study and its SMC language by the density of its populating by ethnically close people.

It is noteworthy, that the historical space of the language existence is, as a rule, not connected with the civilization level of development of its speakers. Languages under study have lived in deep geographic isolation for hundreds (or even thousands) of years. So it would be wrong to consider the language of a more civilized people parent and the language of a less civilized people-descendent and vice versa.

Probably, the best way to define the type of relation between a language under study and its SMC-language is to combine formal method suggested in the present paper and methods of phylogeny. 


\section{Conclusion}

The present research is based on the information from the biggest typological database describing structural properties of languages-WALS. The data were processed using a formal, new for typological studies, method-a method of two-objective optimization.

We introduced the terms: P-pairs of languages (whose genealogic relationship was defined by the methods of comparative linguistics or similar methods based on phonetic data), G-pairs of languages (the relationship was defined by the method of search for SMC-language using structural information from WALS) and PG-pairs (cases when P-pairs and G-pairs match).

A diagram for all languages from WALS that meet the requirements of two-objective optimization was built. It included 523 pairs if languages. We were able to find draw a line through four points separating the area, which includes only genealogically relative languages (PG-pairs), from the area of both P-pairs and G-pairs of languages.

This numerical experiment showed that only $20.4 \%$ of all languages presented in WALS have enough grammar description for being able to classify with their relatives basing on structural information. An important conclusion from this experiment is that grammar features carry important information on the genealogical similarity of the languages.

The formal method aimed at finding SMCL for any language was applied to 19 language isolates that contain enough information in WALS Program. The distribution diagram of pairs "Language isolate \& its SMCL" (Fig. 6) showed that 18 pairs of languages fell below the boundary separating the area of PG-languages, which mean that no conclusion can be made about these languages. Nevertheless, Tiwi and its SMC-languageMaung-fell into the area of genealogically relative languages. It allows us suggest the hypothesis that Tiwi belongs to the Iwaidjan family along with Maung.

The research was also conducted on 35 languages from 25 micro-families that include up to 10 languages and, in our opinion, are similar to language isolates. A SMCL was found for each of the language from microfamilies. The result presented in a diagram showed that no pairs fell in the area of PG-pairs (Fig. 7). That is the reason why we only suggest a hypothesis that if WALS is replenished with more information pairs of languages from micro-families and their SMC-languages will move to the right on the graph.

\section{Acknowledgment}

The paper was checked for the language by Professional editing service (http://www.profediting.com/).

Online Chart Maker (https://live.amcharts.com/) was used for graph visualization.

\section{Funding Information}

The research was supported by RFBR grant No. 1606-00187.

The study was conducted as part of scientificresearch work \#74 within the framework of the basic part of governmental order in the sphere of scientific activity by order $\# 2014 / 113$.

\section{Author's Contributions}

The idea of the study, discussion and conclusion sections and formal description of the method belong to Vladimir Polyakov. The database "Isolates" and the queries were developed by Vladimir Polyakov and Ivan Anisimov. The graphs, calculations and tables were made by Elena Makarova. The authors have equally contributed to the writing of the article.

\section{Ethics}

This article is original and contains unpublished material. The authors confirm that there are no ethical issues involved.

\section{References}

Benveniste, E., 1954. La classification des langues. Conferences de l'Institut de Linguistique de l'Universite de Paris, XI, Annees 1952-1953: 33-50.

Bynon, T., 1977. Historical Linguistics. 1st Edn., Cambridge University Press, Cambridge, ISBN-10: 0521291887, pp: 301.

Codd, E.F., 1970. A relational model of data for large shared data banks. Commun. ACM, 13: 377-87. DOI: $10.1145 / 362384.362685$

Comrie, B., M.S. Dryer, D. Gil and M. Haspelmath, 2013. Introduction. In: The World Atlas of Language Structures Online. Dryer, M.S. and M. Haspelmath (Eds.), Max Planck Institute for Evolutionary Anthropology, Leipzig.

Danilova, V., E. Makarova, V. Polyakov and V. Solovyev, 2016. Frequency-based relevant grammar features of the Caucasian languages. Ind. J. Sci. Technol., 9: 1-10. DOI: $10.17485 / \mathrm{ijst} / 2016 / \mathrm{v} 9 \mathrm{i} 11 / 89415$

Dryer, M.S. and M. Haspelmath, 2013. The world atlas of language structures online. Max Planck Institute for Evolutionary Anthropology, Leipzig.

Edgeworth, F.Y., 1881. Mathematical Psychics. 1st Edn., P. Keagan, London.

Ehrgott, M., 2000. Multicriteria Optimization. 1st Edn., Springer, Berlin, ISBN-10: 3540678697, pp: 243.

Fayyad, U., G. Piatetsky-Shapiro and P. Smyth, 1996. From data mining to knowledge discovery in databases. Ai Magazine, 17: 37-54. DOI: 10.1609/aimag.v17i3.1230 
Gray, R.D., Q.D. Atkinson and S.J. Greenhill, 2011. Language evolution and human history: What a difference a date makes. Philosophical Trans. Royal Society B: Biol. Sci., 366: 1090-1100.

DOI: $10.1098 /$ rstb. 2010.0378

Haspelmath, M., M.S. Dryer, D. Gil and B. Comrie, 2005. The World Atlas of Language Structures. 1st Edn., OUP Oxford, Oxford, ISBN-10: 0199255911, pp: 712 .

Izraylevich, S. and V. Tsudikman, 2012. Automated Option Trading: Create, Optimize and Test Automated Trading Systems. 1st Edn., FT Press, Upper Saddle River, ISBN-10: 0132491907, pp: 304.

Levenshtein, V.I., 1966. Binary codes capable of correcting deletions, insertions and reversals. Soviet Phys. Doklady, 10: 707-710.

Makarova, E. and V. Polyakov, 2015. The origin of the article in Indo-European languages of Western Europe. Mediterranean J. Social Sci., 6: 61-75. DOI: $10.5901 / \mathrm{mjss} .2015 . v 6 n 5 s 4 p 61$
Swadesh, M., 1952. Lexico-statistic dating of prehistoric ethnic contacts: With special reference to North American Indians and Eskimos. Proc. Am. Philosophical Society, 96: 452-463.

Tallerman, M. and K.R. Gibson, 2012. The Oxford Handbook of Language Evolution. OUP Oxford, Oxford, ISBN-10: 0199541116, pp: 763.

Trubetzkoy, N.S., 1939. Gedenken ihrer das Indogermanen Problem, Acta Linguistica. Copenhague, 1: 81-89.

Wichmann, S. and E.W. Holman, 2010. Pairwise Comparisons of Typological Profiles. In: Rethinking Universals: How Rarities Affect Linguistic Theory, Wohlgemuth, J. and M. Cysouw (Eds.), Walter de Gruyter, Berlin, ISBN-10: 311022092X, pp: 241-254.

Wichmann, S., A. Müller, A. Wett, V. Velupillai and J. Bischoffberger et al., 2013. The ASJP Database (version 16). 